\title{
Visual search without central vision - no single pseudofovea location is best
}

\author{
Angelika Lingnau \\ University of Trento \\ Thorsten Albrecht \\ Georg-August-Universität Göttingen \\ Dirk Vorberg \\ Westfälische Wilhems-Universität \\ Münster
}

Jens Schwarzbach

University of Trento

We typically fixate targets such that they are projected onto the fovea for best spatial resolution. Macular degeneration patients often develop fixation strategies such that targets are projected to an intact eccentric part of the retina, called pseudofovea. A longstanding debate concerns which pseudofovea location is optimal for non-foveal vision. We examined how pseudofovea position and eccentricity affect performance in visual search when vision is restricted to an offfoveal retinal region by a gaze-contingent display that dynamically blurs the stimulus except within a small viewing window (forced field location). Trained normally sighted participants were more accurate when forced field location was congruent with the required scan path direction; this contradicts the view that a single pseudofovea location is generally best. Rather, performance depends on the congruence between pseudofovea location and scan path direction.

Keywords: peripheral vision; gaze-contingent display; visual search; central vision

\section{Introduction}

Visual acuity drops fast as one moves from the fovea to the periphery (Abdelnour \& Kalloniatis, 2001). High acuity - as required for tasks such as reading - is restricted to an area of about $1^{\circ}$ around the point of fixation. Therefore, we typically directly fixate important details of our environment, thus projecting relevant information onto the fovea, the retinal region with highest receptor density. This strategy becomes inefficient, however, if the foveal region is damaged, as is the case in macular degeneration (MD). Consequently, MD patients often develop compensatory strategies of moving the eyes to neighbouring locations, such that important visual features of an object are projected to a parafoveal or peripheral rather than the foveal region (see Figure 1).
This region is typically called preferred retinal location (Timberlake et al., 1986) or pseudofovea (Guez, Le Gargasson, \& Rigaudiere, 1993).

\section{Effect of Pseudofovea Location}

How does the particular location of a pseudofovea affect reading performance? Fletcher, Schuchard, \& Watson (1999) studied this question in patients with central scotoma, classified by the location of the pseudofovea they had developed (above, below, left, or right of the central scotoma, as assessed by scanning laser ophthalmoscopy). Mean reading rate did not systematically differ between groups. However, because of substantial variability in reading rate within patient groups, it is difficult to interpret these findings, which points to a general problem in patient research: MD patients typically do not differ in their pseudofovea location only, but in other features as well (e.g. age, 
Journal of Eye Movement Research

$7(2): 4,1-14$
Lingnau, A., Albrecht, T., Schwarzbach, J., \& Vorberg, D. (2014) Visual Search Without Central Vision scotoma size, scotoma location), which makes it difficult to draw firm conclusions from behavioral differences between patient groups. It is therefore not surprising that previous studies using this approach have produced conflicting results concerning which pseudofovea location is suited best for reading (Fletcher, et al., 1999; Nilsson, Frennesson, \& Nilsson, 2003; Petre, Hazel, Fine, \& Rubin, 2000; Stelmack, Massof, \& Stelmack, 2004; Trauzettel-Klosinski \& Brendler, 1998).

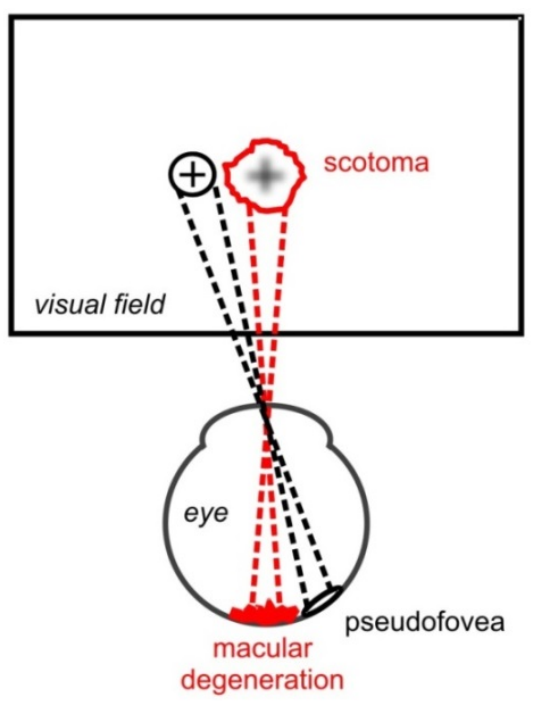

Figure 1: MD patients suffer from a central scotoma due to irreversible damage to the photoreceptors in the macula, the central part of the retina. Consequently, these patients often develop the strategy to fixate a neighbouring location, such that the target is projected onto an intact parafoveal or peripheral location, called pseudofovea.

\section{Eye movements with a Forced Field Location}

Gaze-contingent displays allow to simulate the effects of central visual field loss in normal-sighted participants (e.g. Bertera, 1988; Cornelissen, Bruin, \& Kooijman, 2005; Kwon, Nandy, \& Tjan, 2013; Rayner \& Bertera, 1979; Sommerhalder et al., 2003). We have recently developed a gaze-contingent display technique that dynamically restricts sharp vision to a small region of the visual field relative to fixation (Forced Field Location, FF location) while blurring the remainder of the screen (Lingnau, Schwarzbach, \& Vorberg, 2008, 2010). Using this technique, we have shown that participants who were trained in reading with a particular FF location performance performed best (i.e., needed fewer and shorter fixations per text line) when the FF was to the right of fixation (Lingnau, et al., 2008). Reading performance turned out worst with a FF below fixation, whereas intermediate performance was observed with a FF left of fixation. Under all conditions, eye movement patterns revealed a large proportion of saccades directed towards the forced field location.

Attention and gaze are strongly coupled, with impaired performance if a location has to be attended that does not coincide with the target location of an upcoming saccade (Deubel \& Schneider, 1996; Hoffman \& Subramaniam, 1995). This coupling might also explain the observed performance advantage observed for a right FF location. Reading text from left to right requires gaze shifts to the right (black arrow in Figure 2A and B). With a right $\mathrm{FF}$, participants must shift attention to the right in order to identify letters presented at the FF location (white arrow in Figure 2A). With a FF right of fixation, extraction of visual information at the FF location as well as saccade programming for reading involve attention shifts in the same direction; we refer to this as the congruent condition (see Figure 2A). By contrast, in reading with a $\mathrm{FF}$ to the left of fixation, attention has to be shifted in a direction opposite to gaze direction (Figure $2 \mathrm{~B}$; incongruent condition).

\section{«Reading without central vision»}

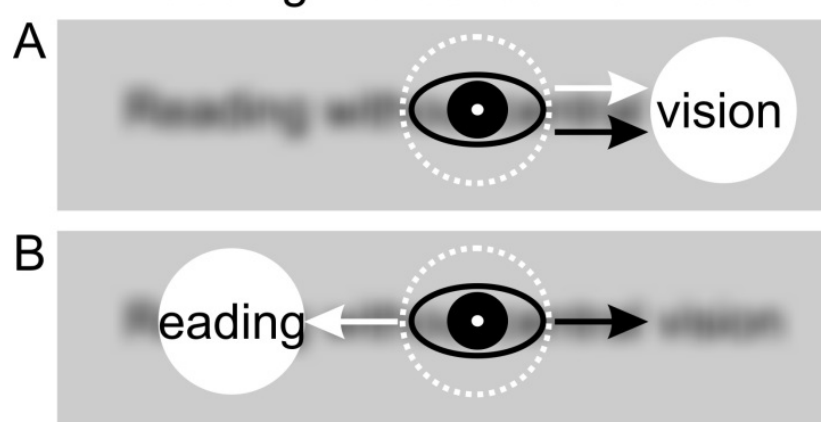

Figure 2: Suggested relationship between gaze and attention. A: If FF location (white disk) is in spatial register with text direction (in the example shown: FF-R), shifts of attention (white arrow) towards the $F F$ and the required direction of eye movements (black arrow) are congruent. Dotted circle: focus of attention; eye symbol: gaze position. B: If FF location is opposite to text direction (in the example shown: FF-L), shifts of attention towards the FF and the required direction of eye movements are incongruent, leading to reduced reading performance as compared to congruent conditions (Lingnau, et al., 2008). FF Location, Forced Field Location; FF-L, FF left of fixation; $\boldsymbol{F F}-\boldsymbol{R}, \mathrm{FF}$ right of fixation. 
Journal of Eye Movement Research

$7(2): 4,1-14$

\section{Aims of the current study}

The goal of the current study was to examine (a) whether the results of our previous study generalize to situations other than reading, and (b) how performance with a particular FF location is affected by FF eccentricity. To this aim, we developed a visual search task that requires sequential eye movements either from left to right, or from right to left (Figure 3). Other than in reading tasks, the stimulus material does not bias a certain preferred direction for visual analysis. We predicted better performance with a FF location that is congruent with scan path direction (e.g., FF location to the right, and scan path left to right) than under incongruent conditions (FF left, scan path direction left to right). To examine how FF eccentricity affects visual search performance, we factorially varied FF eccentricity (near, far) with scan path direction.

\section{Methods}

\section{Participants}

Six female participants, aged between 18 and 30 (mean age: 22.7), took part in the experiment, which consisted of six sessions in total, either as a course requirement or for a compensation of $€ 7,50$ per session. All participants had normal or corrected to normal vision.

\section{Apparatus}

Eye movements from both eyes were recorded by a high-speed $(250 \mathrm{~Hz})$ video-based eye tracking system (Eyelink I). Stimuli were presented on an Iiyama Vision Master 451 monitor (18'), with a screen resolution of 800x600 pixels and a refresh rate of $85 \mathrm{~Hz}$. Viewing distance from the monitor was $76 \mathrm{~cm}$. Gaze contingent stimulus presentation and randomization was programmed in $\mathrm{C}$, using the $\mathrm{MS}$ Visual $\mathrm{C}++6.0$ platform. For eye movement recording and online saccade detection, we used standard libraries supplied with the EyeLink system.

Participants wore a headband with cameras attached. At the beginning of each session, gaze calibration and validation was performed by having participants fixate targets that appeared randomly on a 3 by 3 grid. Calibration was repeated if gaze position differed from
Lingnau, A., Albrecht, T., Schwarzbach, J., \& Vorberg, D. (2014) Visual Search Without Central Vision

the initial calibration measurements by more than 0.5 degrees.

\section{Gaze-contingent display procedure}

We used a gaze-contingent display procedure (Lingnau et al., 2008; 2010) which presents the stimulus such that it is blurred everywhere except at a small circular area, the forced field location. The forced field location moves with the eye, which is monitored by a video-based eye tracking system (EyeLink I, SR Research). For each trial, two bitmaps are created offline, one containing the original visual search display ('Sharp'; Figure 3A), and one containing a blurred version ('Blur'; Figure 3B) of the original stimulus by smoothing with a Gaussian filter. At the beginning of the trial, Blur is shown. After each eye movement, the intensity values within a 81 x 81 pixel region (corresponding to $2.41 \times 2.41^{\circ}$ ) around the current $\mathrm{FF}$ location in Blur and Sharp are averaged, weighted according to a weight matrix $\omega(\mathrm{i}, \mathrm{j})$ that defines the extent and shape of the FF location, with smooth transitions at the boundaries. The matrix containing the averaged intensity values at the FF location is copied to the screen. For technical details, see Lingnau et al. (2008).

\section{Task}

On each trial, participants had to scan a search display which consisted of boxes positioned on a horizontal line (see Figure 3A), and to count the number of boxes with center of gravity above the line. Scan-path direction was enforced by having participants start each trial from the small red square located either at the left or the right end of the line.

\section{Design}

Forced field location (left, right), FF eccentricity (near: $1.81 \%$ far: $2.71^{\circ}$ ) and scan path direction (left-toright, LR; right-to-left, RL) were combined factorially as independent variables. The conditions FF left of fixation (FF-L) and $F F$ right of fixation (FF-R) were defined by the center coordinates of the FF relative to fixation (FF-L, near: $-1.81^{\circ} / 0^{\circ}$, far: $-2.71 \%{ }^{\circ}$; FF-R, near: $1.81^{\circ} / 0^{\circ}$, far: $\left.2.71^{\circ} / 0^{\circ}\right)$. FF location and $\mathrm{FF}$ eccentricity remained constant within but varied between trial blocks, whereas scan path direction randomly varied from trial to trial. 
A

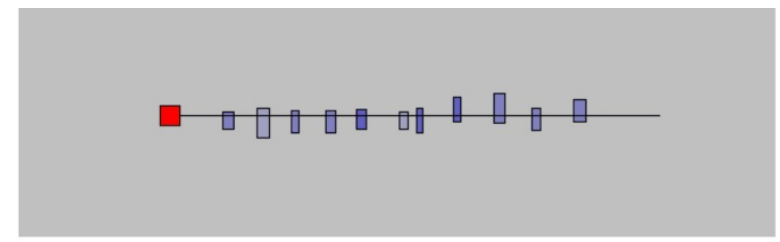

B
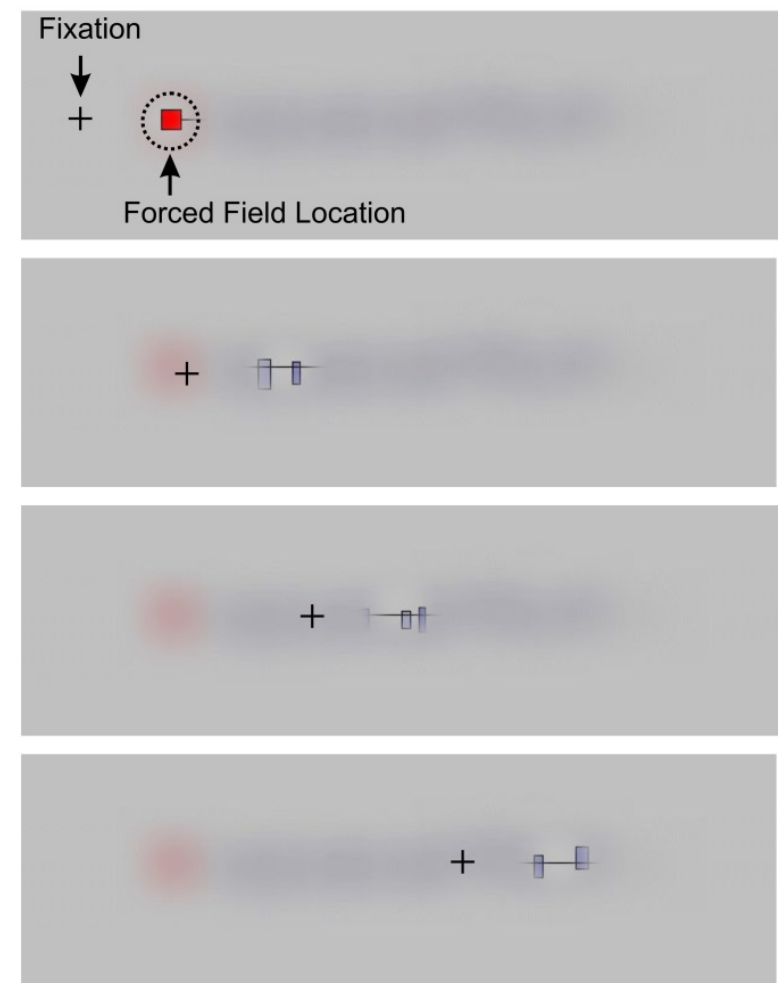

Figure 3: Stimuli and experimental procedure. A: Clear version of an example stimulus. The task is to determine the number of boxes with center of gravity above the horizontal line (in the example shown: 3 boxes), starting from the red rectangle location. B: The gaze-contingent display blurs the display except at a small viewing window (the forced field location) with constant offset with respect to fixation (for details of the procedure, see Lingnau et al., 2008). In the figure, the cross indicates hypothetical fixation position, which was not shown during the experiment.

\section{Materials}

Each stimulus contained 10 to 14 blue boxes (luminance: 14.28-36.96 cd/ m², CIExy: 0.245, 0.203 $0.318,0.318$ ) placed on a horizontal line subtending $12.02^{\circ}$ (see Figure 3A), presented on a uniform gray background (luminance: $53.23 \mathrm{~cd} / \mathrm{m}^{2}$, CIExy: 0.346, 0.359 ). Box width varied between 0.15 and $0.33^{\circ}$, box height between 0.42 and $0.78^{\circ}$, with distance between adjacent boxes between 0.18 and $1.03^{\circ}$. Boxes were
Lingnau, A., Albrecht, T., Schwarzbach, J., \& Vorberg, D. (2014) Visual Search Without Central Vision

randomly jittered upwards or downwards by $1 / 4$ or $1 / 6$ of their height. A red rectangle (luminance: $22.27 \mathrm{~cd} / \mathrm{m}^{2}$; CIExy: $0.649,0.331$ ) at the left or right end of the line indicated the start position. To prevent participants from solving the task on the basis of the absolute stimulus position on the screen, the horizontal reference line was located randomly either $2.11^{\circ}$ above or below fixation.

\section{Trial procedure}

Participants started a trial by pressing a button while fixating a dot on the centre of the screen, after which three single dots on a horizontal line were presented for 6 seconds (secs), followed by the blurred version of the stimulus. The location of the red square indicated the start position and thus the direction in which to search the display. As soon as participants felt sure about the number of boxes presented above the line, they pressed a button and pronounced their response. In the following, we refer to the total time till button press as the search time. The trial terminated automatically if no button press was given within 60 seconds.

\section{Instruction}

At the beginning of the first session, general information was given about eye tracking, stressing the importance of avoiding head and body movements during data recording. Participants were instructed to move the jaw as little as possible when speaking the response (number of boxes above line). Before each block, participants were informed whether the FF location in the upcoming block was to the left or to the right of fixation.

\section{Layout of experimental sessions}

Participants performed six sessions consisting of eight blocks each. Each block consisted of 10 trials. The first session served to familiarize participants with the experimental procedure and with searching with a FF. Excluding the first session, there were 50 replications per condition and participant.

\section{Data analysis}

We used standard Eyelink I libraries for detection of saccades and fixations. Saccades were identified either when velocity exceeded $30 \%$ or acceleration exceeded $8000 \%$ s. Offscreen fixations and blinks were removed from the data. We used the FF positions, rather than the positions of central fixation, for analysis. Thus, we refer 
Journal of Eye Movement Research

$7(2): 4,1-14$

to the landing positions of the FF center coordinates as fixations. Search time, number of fixations and fixation durations were computed collapsing correct and incorrect trials.

\section{Results}

\section{Visual Search Performance}

Mean number of fixations, fixation duration, search time and percentage of errors (see Tables 1-4) were subjected to separate $5 \times 2 \times 2 \times 2$ repeated-measures ANOVAs, with session, FF eccentricity, FF location and scan path direction as factors. Degrees of freedom were adjusted by the Huyn-Feldt procedure when appropriate (associated p-values are denoted as pHF).

Training effects. Figure 4 (left panel) shows that performance improved throughout experimental sessions, as indicated by decreases in search time, number and duration of fixations, and percentage of errors. These observations are corroborated by statistical analyses, as revealed by a main effect of training session for search time $[\mathrm{F}(4,20)=10.48, \mathrm{p}<.0001$; session 2: 22.7 seconds $(\mathrm{sec})$, session 6: $13.2 \mathrm{sec}$, number of fixations $\left[\mathrm{F}(4,20)=3.69, \mathrm{p}_{\mathrm{HF}}=.061\right.$; session $2: 54.8$, session $\left.6: 40.7\right]$, fixation duration $[\mathrm{F}(4,20)=8.64, \mathrm{p}<.0001$; session $2: 603$ milliseconds (msec), session 6: $430 \mathrm{msec}]$ and percentage of errors $[F(4,20)=9.77, \mathrm{p}<.0001$; session $2: 45.9$, session 6: 24.2].

Eccentricity. Search time, number of fixations, fixation duration, and percentage of errors increased with FF eccentricity (Figure 4, middle panel), as evidenced by the main effect of FF eccentricity for search time $[\mathrm{F}(1,5)=16.97, \mathrm{p}=.009$; near: $13.6 \mathrm{sec}$, far: $20.5 \mathrm{sec}]$, number of fixations $[F(1,5)=7.07, p=.045$; near: 40.1, far: 53.1], fixation duration $[\mathrm{F}(1,5)=14.75, \mathrm{p}=.012$; near: 469 $\mathrm{msec}$, far: $516 \mathrm{msec}$, and percentage of errors $[\mathrm{F}(1,5)=14.06, \mathrm{p}=.013$, near: $24.5 \%$, far: $36.5 \%$ ].

Forced field location. There was no significant overall main effect of FF location (L vs. R) on search time $[\mathrm{F}(1,5)=.996, \mathrm{p}=.364]$, number of fixations, $[\mathrm{F}(1,5)=.492, \mathrm{p}=.514]$, fixation duration $[\mathrm{F}(1,5)=.051$, $\mathrm{p}=.831]$, or percentage of errors $[\mathrm{F}(1,5)=.176, \mathrm{p}=.693]$.
Lingnau, A., Albrecht, T., Schwarzbach, J., \& Vorberg, D. (2014) Visual Search Without Central Vision

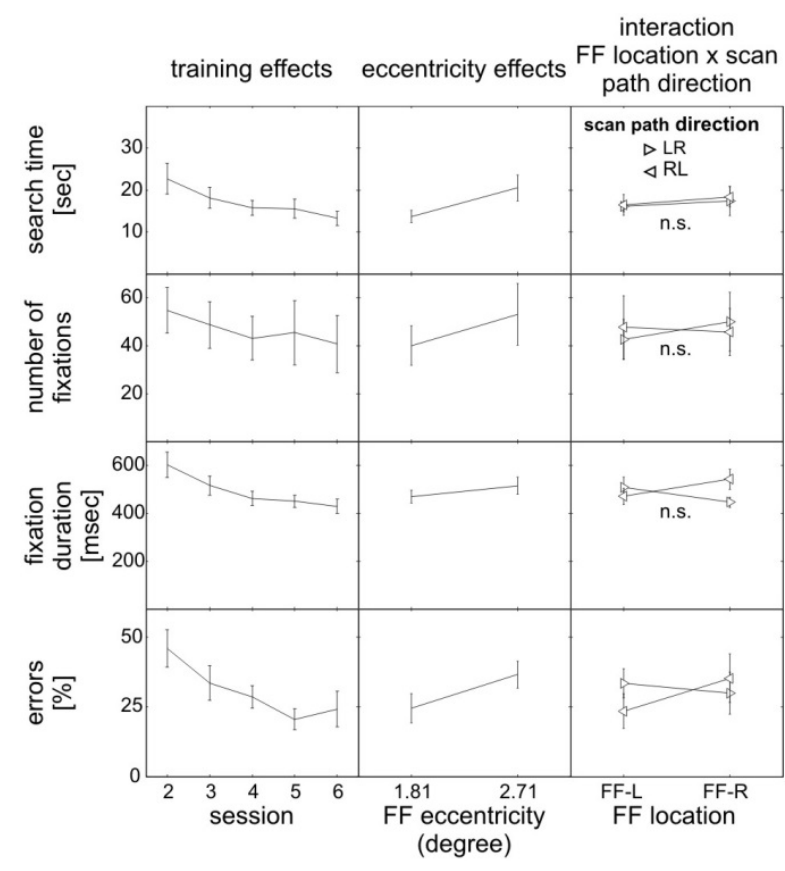

Figure 4: Main effects of session (left column), FF eccentricity (middle column) and interaction of FF location $\times$ scan path direction (right column) on search time, number of fixations, fixation duration, and percentage errors. FF, Forced Field; $\boldsymbol{F F}-\boldsymbol{L}, F F$ left of fixation; $\boldsymbol{F F}-\boldsymbol{R}, F F$ right of fixation; $\boldsymbol{L R}$, left to right; $\boldsymbol{R L}$, right to left. Error bars indicate standard error of the mean.

Scan path direction. Scan path direction had no significant effect on search time $[\mathrm{F}(1,5)=.888, \mathrm{p}=.389]$, number of fixations $[\mathrm{F}(1,5)=.024, \mathrm{p}=.882]$, fixation duration $[\mathrm{F}(1,5)=4.39, \mathrm{p}=.09]$, or percentage of errors $[\mathrm{F}(1,5)=.830, \mathrm{p}=.404]$.

Interaction FF location $\times$ scan path direction. Figure 4 (right panel) shows that participants produced less errors and made slightly shorter fixations when FF location and scan path direction were congruent. This observation is supported by the reliable interaction between FF location and scan path direction for percentage of errors $[F(1,5)=9.824, p=.026]$ and by the corresponding trend towards an interaction for fixation duration $[\mathrm{F}(1,5)=5.99, \quad \mathrm{p}=.058]$. No significant interactions were found for search time $[F(1,5)=.049$, $\mathrm{p}=.833]$ and number of fixations $[\mathrm{F}(1,5)=.774, \mathrm{p}=.419]$. None of the other interactions was significant (all $\mathrm{p}>$ $.05)$. 
Journal of Eye Movement Research $7(2): 4,1-14$

\section{Eye movement patterns}

The top panel of Figure 5 shows the frequency distributions of the horizontal saccade amplitudes, for near (column 2 and 3) and for far (column 1 and 4) FF eccentricity on congruent trials, separately for each participant (columns 1 and 2: FF-L, scan path direction right to left, columns 3 and 4: FF-R, scan path direction left to right; FF locations indicated by grey bar). The distributions typically consist of three components, two of them sharply peaked, and one with large variance. As clearly seen, most saccades were directed along the required scan path (i.e. towards the FF location), as evicenced by the narrow distribution centered in the middle or at the border of the FF location in the majority of cases. The other two distributions come from saccades opposite to the scan path direction (i.e. away from the FF location).

On incongruent trials (Figure 5, bottom panel), most saccades were performed in the required scan path direction (i.e., away from the FF location), with fewer saccades directed towards the FF (i.e., opposite to the scan path direction).

To quantify the effect of FF eccentricity on saccade amplitude, we fitted three-component Gaussian mixture distributions to the distribution of horizontal saccade amplitudes separately for each condition and participant (see Figure 6 for an illustration), using an adapted version of the gaussmix function of the MATLAB 5.3 voicebox toolbox. This method estimates the mean $(\mu)$, standard deviation $(\sigma)$ and weight $(\omega)$ of each component distribution.

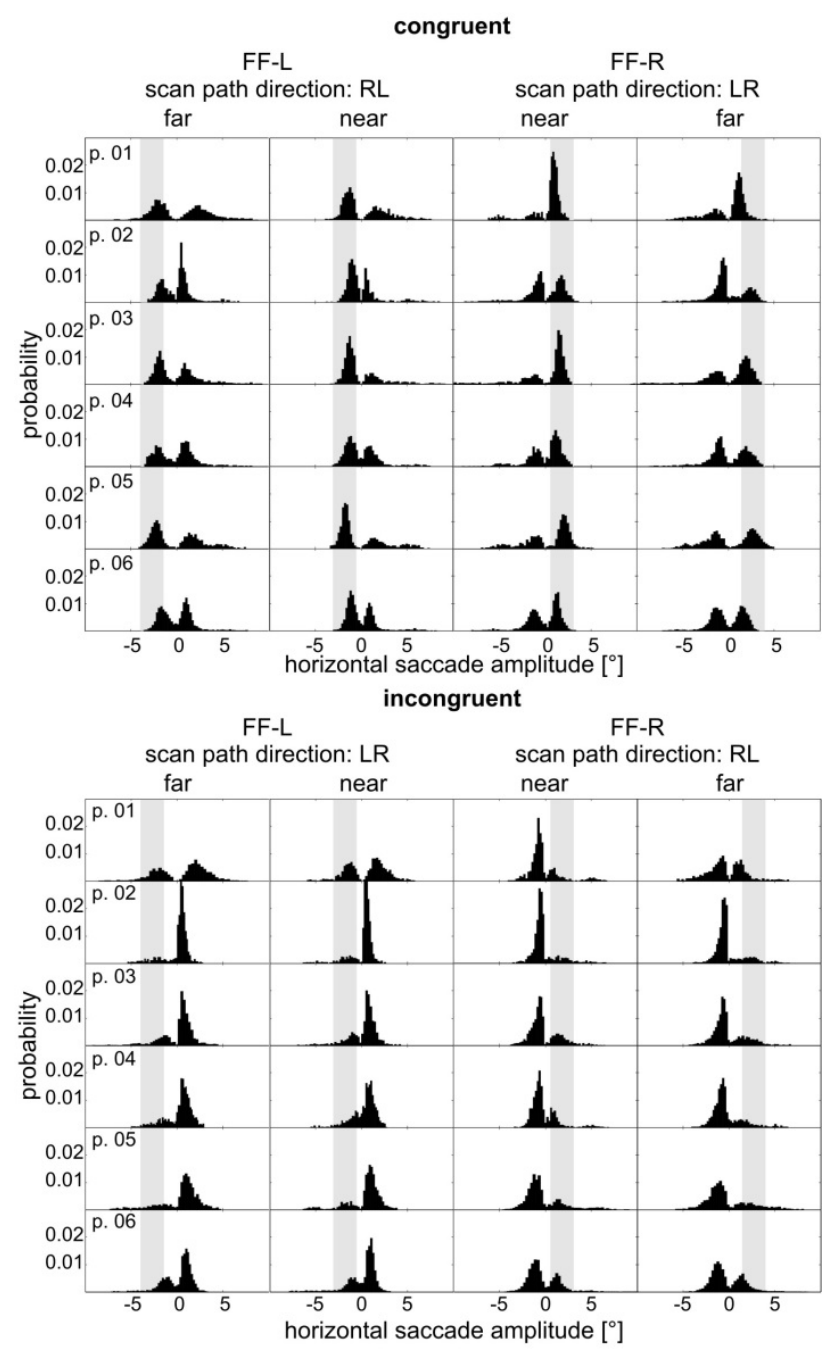

Figure 5: Frequency distributions of horizontal saccade amplitudes, on congruent (top panel) and on incongruent (bottom panel) trials, for FF location at the near (columns 2 and 3) and far (columns 1 and 4) eccentricity, separately for each participant. Grey bars indicate the forced field location. Participants are sorted according to their mean performance in terms of number of fixations (participant 1: best). FF, Forced Field; FF-L, FF left of fixation; FF-R, FF right of fixation; $\boldsymbol{L} \boldsymbol{R}$, left to right; $\boldsymbol{R} \boldsymbol{L}$, right to left. 


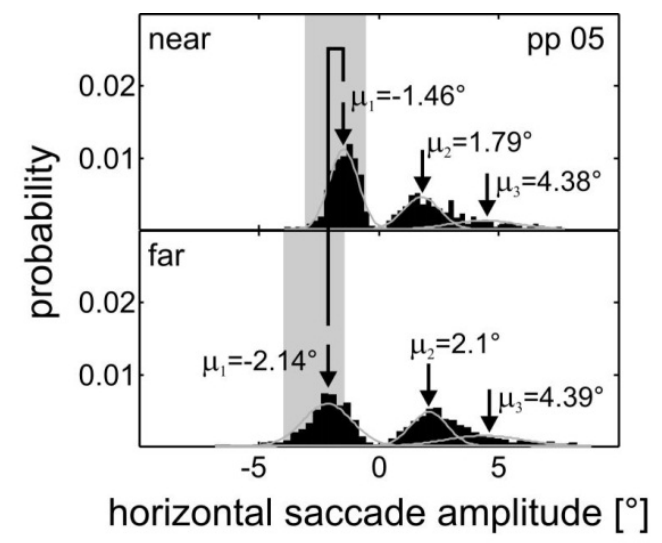

Figure 6: Example results of fitting three-component Gaussian mixture distributions (example: FF-L, scan path direction: $R L$ ). Component distributions (gray lines) and estimated means are highlighted on top of the histrogram. The critical comparison concerns the component distributions of saccades towards FF location (indicated by grey bars).

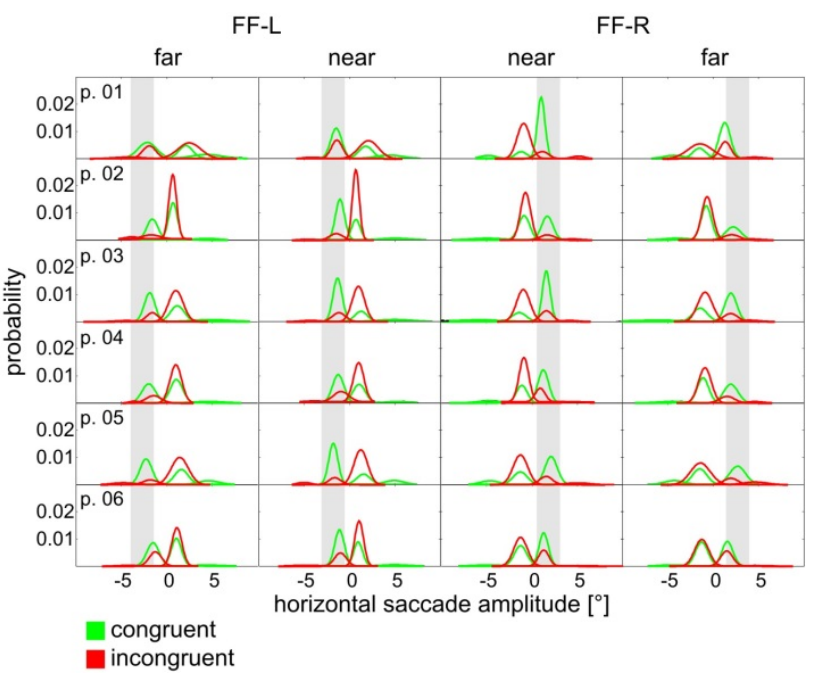

Figure 7: Estimated horizontal saccade amplitude distributions on congruent (green) and incongruent (red) trials, for FF locations at near (columns 2 and 3) and far eccentricity (columns 1 and 4), separately for each participant. Grey bars indicate forced field location. FF, Forced Field; FF-L, FF left of fixation; $\boldsymbol{F F}-\boldsymbol{R}$, FF right of fixation.

The resulting component distributions are shown in Figure 7. On congruent trials (green curves), the majority of saccades were directed towards the FF, whereas on incongruent trials, most saccades went in the opposite direction, in line with the observations made in Figure 6. Thus, participants tended to saccade in the direction required by the task, which coincided with the attention shift required by the FF location on congruent, but was
Lingnau, A., Albrecht, T., Schwarzbach, J., \& Vorberg, D. (2014) Visual Search Without Central Vision

incompatible on incongruent trials. Moreover, saccade amplitudes towards the FF increase with FF eccentricity, as a comparison of the near and far conditions in Figure 8 shows.

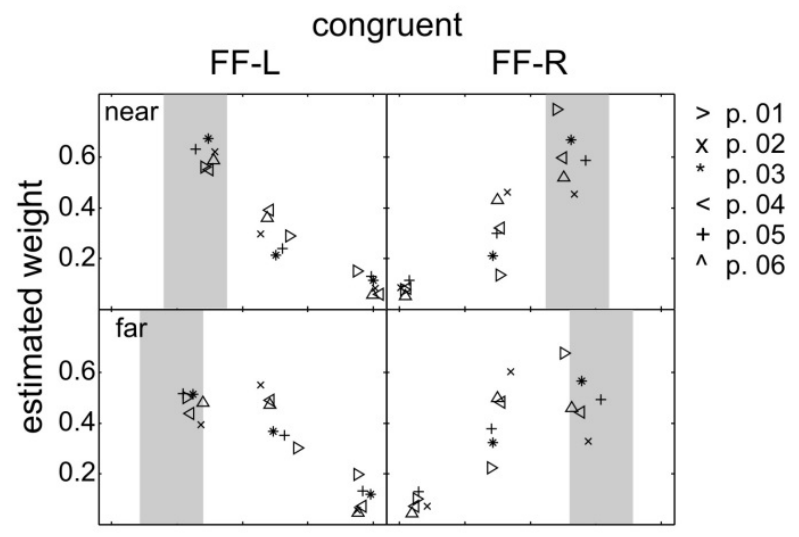

incongruent

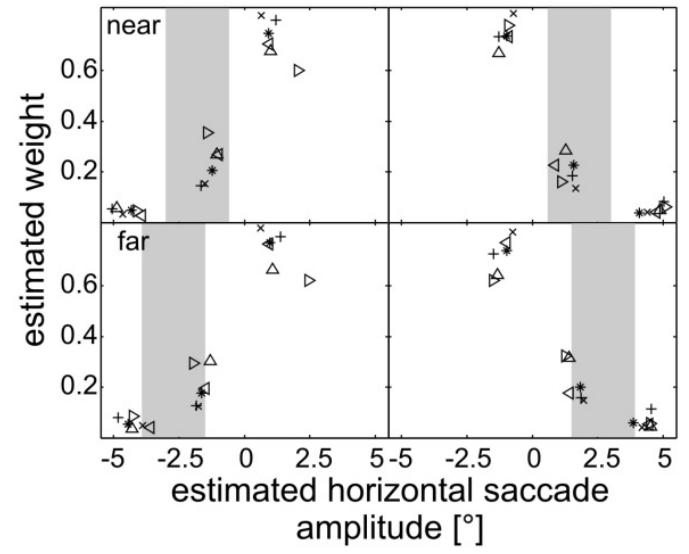

Figure 8. Top panel: Estimated means and weights of the component distributions, for near (upper panel) and far (lower panel) FF eccentricity, on congruent trials. Left panel: FF-L, scan path direction: $R L$, right panel: $F F-R$, scan path direction: $L R)$. As can be seen, a large amount of saccades were directed to the border of the FF location. A comparison between the upper and the lower panel shows the effect of FF eccentricity on horizontal saccade amplitude. Bottom panel: Same as top panel, on incongruent trials. FF, Forced Field; FF-L, FF left of fixation; $\boldsymbol{F F}-\boldsymbol{R}, \mathrm{FF}$ right of fixation.

Figure 9 summarizes the estimates of the means and weights of the horizontal saccade amplitude distributions, on congruent (top panel) and on incongruent (bottom panel) trials. As in Figures 5 and 7, a large proportion of saccades went towards the FF location. Furthermore, a comparison between near and far eccentricity conditions indicates that horizontal saccade amplitude towards the 
FF increases with FF eccentricity. A closer look at this effect is given in Figure 9, showing the estimated horizontal saccade amplitude of saccades towards the FF location versus FF eccentricity. To give an impression of the size of these effects, the dashed line indicates the location of the FF centre: if modulation of horizontal saccade amplitude by the eccentricity of the FF were perfect, the lines for individual participants should be parallel to this "ideal" line. As clearly seen, mean saccade amplitude increases with eccentricity for all participants, though less steeply than predicted from perfect adjustment.

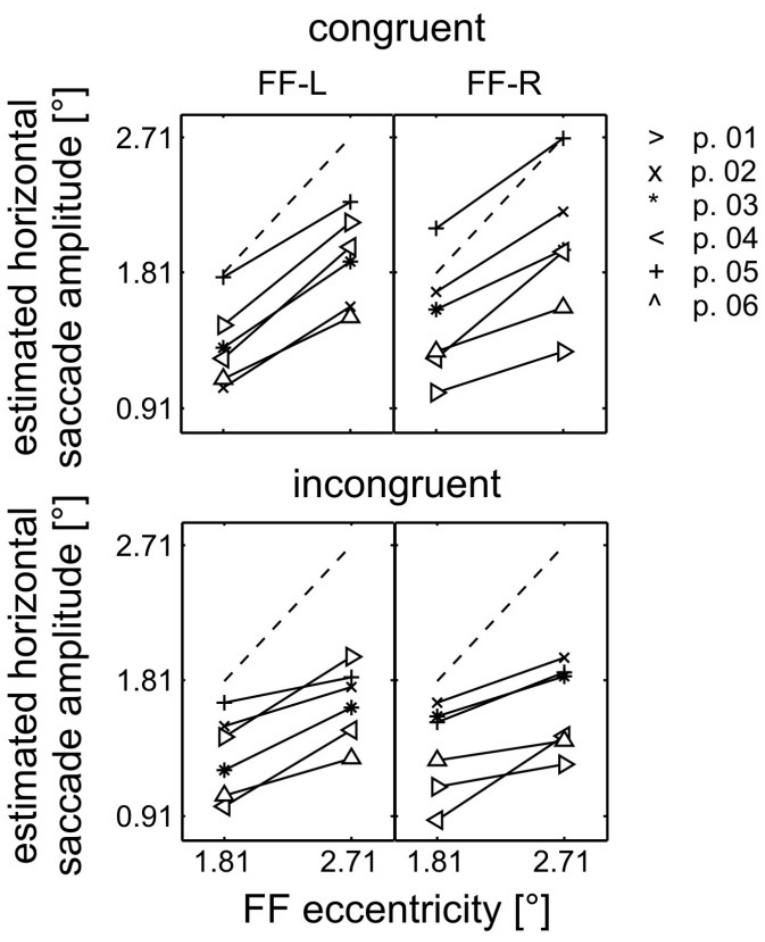

Figure 9: Effects of FF eccentricity on horizontal saccade amplitude, for congruent trials. Top, left panel: FF-L, scan path direction: RL, right panel: $F F-R$, scan path direction: $L R$. Each individual line shows the estimates of horizontal saccade amplitude towards the FF location against FF eccentricity. Dotted lines indicate an ideal line if adjustment of horizontal saccade amplitude were perfect. Bottom: same, for incongruent trials. FF, Forced Field; FF-L, FF left of fixation; FF-R, FF right of fixation; $\boldsymbol{L} \boldsymbol{R}$, left to right; $\boldsymbol{R} \boldsymbol{L}$, right to left.

To quantify the observation in Figure 9 that the eccentricity effects reflect an influence of the FF location that is stronger on congruent than on incongruent trials, we computed a $2 \times 2 \times 2$ repeated-measures ANOVA of estimated horizontal saccade amplitude, with FF eccentricity, FF location, and scan path direction as factors. There was a significant main effect of FF eccentricity $[\mathrm{F}(1,5)=79.92, \quad \mathrm{p}<.0001 ;$ mean saccade amplitude near: $1.36^{\circ}$, far: $1.78^{\circ}$. Furthermore, there were reliable interactions of scan path direction and FF eccentricity $[\mathrm{F}(1,5)=11.64, \mathrm{p}=.019]$, as well as of $\mathrm{FF}$ location, scan path direction, and FF eccentricity $[F(1,5)=56.02, p=.001]$. These findings support the conclusion that horizontal saccade amplitude increases with FF eccentricity, and that this modulation depends on the congruence of FF location with scan path direction.

\section{Discussion}

\section{Coupling of gaze and attention}

Our participants performed a horizontal visual search task in which clear vision was limited to a small viewing window, the forced field FF, which was diplaced from fixation either along with (congruently) or opposite to (incongruently) the search direction. We found that participants made more errors and tended to require longer fixations on incongruent condition compared to congruent trials.

Our results are compatible with the view that, in the absence of central vision, successful performance in reading and visual search strongly depends on the coupling between gaze and attention, with superior performance when gaze and attention are in spatial register (Lingnau, et al., 2008, 2010). More specifically, we suggest that, in order to process information provided at the FF location, participants have to transiently shift visual attention away from fixation and towards the FF location. At the same time, in order to read or to perform visual search, participants have to plan and execute eye movements in the direction determined by the task. Moreover, we argue that performance is likely to be better if the direction of the transient shift of attention towards the FF location is spatially congruent with that of the upcoming eye movement, as compared to conditions that require attention and gaze to be directed in opposite directions.

There are several physiological mechanisms that might underly the congruence effects we observed. It is possible that the conflict arises at the level of spatial attention. We assume that both the processing of visual information at the FF location and the planning and 
execution of eye movements in the scan path direction require transient shifts of attention. Thus the results we obtained would be due to conflicting shifts of attention towards the FF location and shifts of attention in task direction on incongruent trials. Alternatively, it is possible that the origin of conflict we observed is a conflict between the direction of attention shifts and that of eye movements. Assuming that both attention shifts and eye movements share overlapping neuronal resources (Corbetta et al., 1998; Moore \& Armstrong, 2003; but see Thompson, Biscoe, \& Sato, 2005), it is possible that shifting attention opposite to gaze direction results in the conflicts here reported (Deubel \& Schneider, 1996; Hoffman \& Subramaniam, 1995; but see Montagnini \& Castet, 2007).

Note that such a conflict does not allow us to distinguish whether the planning of an eye movement is a necessary precondition of the allocation of spatial attention, as predicted by the premotor theory of attention (Rizzolatti, Riggio, Dascola, \& Umilta, 1987), or whether allocation of attention is required for action preparation, as argued within the Selection for Action (SfA) framework (Schneider, 1995; Schneider \& Deubel, 2002; for a recent review, see Smith \& Schenk, 2012). To us, conflicts between gaze direction and attention seem equally plausible if one assumes that they are implemented in overlapping but independent neuronal substrates, such as the frontal eye fields (Thompson, et al., 2005) or the superior colliculus (Sparks, 1999), structures that are known to play a role in orienting and saccade triggering

Both the frontal eye fields and the superior colliculus have been suggested to contain salience maps for representing potential targets, with the point of highest salience determining the saccade target (Findlay \& Walker, 1999). In our experimental search paradigm, both the upcoming path along the visual stimulus and the FF location are likely to lead to peaks on such salience maps. If the peak corresponding to the FF location coincides with the peak corresponding to the upcoming path on the search stimulus, no conflict arises. By contrast, if the peak related to the FF location is distant from the peak related to the visual search stimulus (on incongruent trials: in the opposite direction), a conflict arises which resuls in impaired performance.

\section{Eccentricity effects}

We observed that search time, number of fixations, fixation duration and percentage of errors increase with FF eccentricity. These results are in line with previous studies reporting that fixation duration increases with scotoma size (Cornelissen, et al., 2005).

Our analyses of the eye movement patterns revealed that horizontal saccade amplitude in the direction of the FF location increases with FF eccentricity. The degree of modulation depends on the congruence between FF location (left vs. right) and scan path direction (LR vs. $\mathrm{RL}$ ), with stronger modulation under congruent than incongruent conditions. In other words, horizontal saccade amplitude matches FF eccentricity more closely on congruent than on incongruent trials.

A possible explanation of this finding is that information provided at the FF location attracts attention, and that this effect is stronger for near than for far eccentricities (Seiple, Clemens, Greenstein, Holopigian, \& Zhang, 2002). On congruent trials, FF location was in spatial register with upcoming gaze shifts, thus facilitating saccades towards the FF, leading to horizontal saccade amplitudes that closely match FF eccentricity. By contrast, on incongruent trials, participants had to shift attention towards the FF location but withhold a saccade towards it. We hypothesize that under these circumstances, the overt "attraction" of gaze towards the FF location is weaker due to an active suppresion of saccades in the wrong direction.

\section{Practical considerations}

Macular degeneration is the leading cause of blindness in developed countries (Friedman et al., 2004). Though progress has been achieved on the medical treatment of macular degeneration, treatment currently focuses on the prevention of the progression of the disease and on the symptoms that develop in advanced stages (Chakravarthy, Evans, \& Rosenfeld, 2010; Rattner \& Nathans, 2006). Consequently, improving visual skills by means of effective training programs is an important goal for these patients.

Performance in reading (Legge, Rubin, Pelli, \& Schleske, 1985) and in visual search (Coeckelbergh, Brouwer, Cornelissen, \& Kooijman, 2002; Cornelissen, et al., 2005; Kwon et al., 2012) is severely reduced, both in patients with central visual field loss and in normal 
sighted participants with simulated central visual field loss. Understanding the mechanisms underlying this severe impairment is a precondition for developing efficient rehabilitation programs/tools.

Reduced visual acuity in the periphery is clearly one but not the only reason for reduced reading performance in central visual field loss, as reading rates in the periphery remain lower than in the central visual field even if character size is enlarged appropriately (Latham \& Whitaker, 1996). Another important factor that has been discussed in the past is poor oculomotor control in patients with central visual field loss. In particular, these patients have to learn to use eccentric fixation, i.e. to position gaze at a constant off-foveal location that projects the target to unimpaired retinal locations. Oculomotor training has been shown to improve reading speed in MD (Seiple, Szlyk, McMahon, Pulido, \& Fishman, 2005) and in hemianopic patients (Schuett Heywood, Kentridge, and Zihl (2008).

It has been suggested that patients might profit from explicit information regarding their current gaze position with respect to the target by using secondary visual feedback, which has been shown to help participants establish eccentric fixation (Zeevi, Peli, \& Stark, 1979). Kwon et al. (2013) recently demonstrated that, displaying a fixation cross at the desired eccentric retinal location and instructing participants to follow a target with this "gaze marker", enables normal-sighted participants to adapt oculomotor control within 15 to 25 hours of training such that the precision with which they reach the the landing site of the first saccade towards the desired eccentric location reaches that of control participants.

In the present study, increasing FF eccentricity by about $50 \%$ caused a $51 \%$ increase in search time and a $49 \%$ increase in number of errors; number of fixations increased by $32 \%$, whereas fixation duration increased by $10 \%$. These dramatic performance deteriorations are line with findings reported by Latham and Whitaker (1996) and Legge et al. (1985). In view of such strong dependence of performance on eccentricity, we predict that MD patients will benefit most from training programs taylored to the individual performance level, starting with a FF at a near eccentricity (as is often the case in an early stage of the disease), increasing eccentricity step by step whenever performance improves. By providing patients the opportunity to start with a task they can solve and which becomes more demanding gradually, we expect such adaptive training programs to provide the motivation for patients to keep on, and thus to reach higher and more stable individual performance levels than current training programs do. Further experiments, possibly in combination with secondary visual feedback training (Kwon, et al., 2013; Zangemeister, Dannheim, \& Kunze, 1986; Zeevi, et al., 1979), will be needed to prove the benefits of such an approach.

Our study suggests that one important factor likely to affect performance in central visual field loss is the coordination between shifts of attention and gaze, at least in the early stages when the oculomotor reference is known to remain at fixation (White \& Bedell, 1990). The results reported by Kwon et al. (2013) suggest that the shift of the oculomotor reference towards an eccentric fixation can be facilitated by secondary visual feedback.

Our participants showed substantial improvements in their ability to perform exclusively on the basis of parafoveal vision, managing to reduce search time by about $70 \%$ within five hours of training. We expect that the gaze-contingent window procedure used in the current study also to be helpful for establishing a pseudofovea in MD patients, even within early phases of their visual disability. Such a training in the beginning of the disease seems promising, in view of the observation that it is easier to establish an appropriate pseudofovea location by proper training, before one has been formed without guidance at a less favorable location (Nilsson, Frennesson, \& Nilsson, 1998).

\section{Conclusions}

Our findings demonstrate that performance in peripheral viewing strongly depends on the congruence between the forced field location and the direction of the scan path, and thus may require dynamical switching between several different retinal locations for different perceptual tasks. In line with our previous results, the current study shows that there is no single pseudofovea location that is optimal in general. Rather, our data suggest that performance in reading and visual search without central vision depends on the congruence between the direction of attentional shifts towards the pseudofovea and the scan path direction required by the task at hand. 
Journal of Eye Movement Research

$7(2): 4,1-14$
Lingnau, A., Albrecht, T., Schwarzbach, J., \& Vorberg, D. (2014) Visual Search Without Central Vision

\section{Acknowledgements}

This research was supported by a grant from the Studienstiftung des deutschen Volkes (German National Merit Foundation) to A.L., and by the Provincia di Autonoma di Trento and the Fondazione Cassa di Risparmio di Trento e Rovereto.

\section{References}

Abdelnour, O., \& Kalloniatis, M. (2001). Word acuity threshold as a function of contrast and retinal eccentricity. Optometry and Vision Science, 78, 914-919.

Bertera, J. H. (1988). The effect of simulated scotomas on visual search in normal subjects. Invest Ophthalmol Vis Sci, 29(3), 470-475.

Chakravarthy, U., Evans, J., \& Rosenfeld, P. J. (2010). Age related macular degeneration. [Review]. BMJ, 340, c981.

Coeckelbergh, T. R. M., Brouwer, W. H., Cornelissen, F. W., \& Kooijman, A. C. (2002). The effect of visual field defects on eye movements and practical fitness to drive. Vision Research, 42, 669-677.

Corbetta, M., Akbudak, E., Conturo, T. E., Snyder, A. Z., Ollinger, J. M., Drury, H. A., et al. (1998). A common network of functional areas for attention and eye movements. Neuron, 21(4), 761-773.

Cornelissen, F. W., Bruin, K. J., \& Kooijman, A. C. (2005). The influence of artificial scotomas on eye movements during visual search. Optometry and Vision Science, 82(1), 27-35.

Deubel, H., \& Schneider, W. X. (1996). Saccade target selection and object recognition: evidence for a common attentional mechanism. Vision Research, 36, 1827-1837.

Findlay, J. M., \& Walker, R. (1999). A model of saccade generation based on parallel processing and competitive inhibition. Behavioral and Brain Sciences, 22, 661-721.
Fletcher, D. C., Schuchard, R. A., \& Watson, G. (1999). Relative locations of macular scotomas near the PRL: effect on low vision reading. Journal of Rehabilitation Research and Development, 36(4), 356-364.

Friedman, D. S., O'Colmain, B. J., Munoz, B., Tomany, S. C., McCarty, C., de Jong, P. T., et al. (2004). Prevalence of age-related macular degeneration in the United States. Archives of Ophthalmology, 122(4), 564-572.

Guez, J. E., Le Gargasson, J. F., \& Rigaudiere, F. (1993). Is there a systematic location for the pseudofovea in patients with central scotoma? Vision Research, 33, 1271-1279.

Hoffman, J. E., \& Subramaniam, B. (1995). The role of visual attention in saccadic eye movements. Perception \& Psychophysics, 57, 787-795.

Kwon, M., Nandy, A. S., \& Tjan, B. S. (2013). Rapid and persistent adaptability of human oculomotor control in response to simulated central vision loss. Current Biology, 23(17), 1663-1669.

Kwon, M., Ramachandra, C., Satgunam, P., Mel, B. W., Peli, E., \& Tjan, B. S. (2012). Contour enhancement benefits older adults with simulated central field loss. Optometry and Vision Science, 89(9), 1374-1384.

Latham, K., \& Whitaker, D. (1996). A comparison of word recognition and reading performance in foveal and peripheral vision. Vision Research, $36,2665-2674$.

Legge, G. E., Rubin, G. S., Pelli, D. G., \& Schleske, M. M. (1985). Psychophysics of reading - II. Low Vision. Vision Research, 25, 253-266.

Lingnau, A., Schwarzbach, J., \& Vorberg, D. (2008). Adaptive strategies for reading with a forced retinal location. Journal of Vision, 8(5), 6 1-18.

Lingnau, A., Schwarzbach, J., \& Vorberg, D. (2010). (Un-) coupling gaze and attention outside central vision. Journal of Vision, 10(11), 1-13.

Montagnini, A., \& Castet, E. (2007). Spatiotemporal dynamics of visual attention during saccade preparation: Independence and coupling between attention and movement planning. Journal of Vision, 7(14), 8 1-16. 
Journal of Eye Movement Research

$7(2): 4,1-14$

Moore, T., \& Armstrong, K. M. (2003). Selective gating of visual signals by microstimulation of frontal cortex. Nature, 421(6921), 370-373.

Nilsson, U. L., Frennesson, C., \& Nilsson, S. E. G. (1998). Location and stability of a newly established eccentric retinal locus suitable for reading, achieved through training of patients with a dense central scotoma. Optometry and Vision Science, 75, 873-878.

Nilsson, U. L., Frennesson, C., \& Nilsson, S. E. G. (2003). Patients with AMD and a large absolute central scotoma can be trained successfully to use eccentric viewing, as demonstrated in a scanning laser ophthalmoscope. Vision Research, 43(16), 1777-1787.

Petre, K. L., Hazel, C. A., Fine, E. M., \& Rubin, G. S. (2000). Reading with eccentric fixation is faster in inferior visual field than in left visual field. Optometry and Vision Science, 77(1), 34-39.

Rattner, A., \& Nathans, J. (2006). Macular degeneration: recent advances and therapeutic opportunities. Nature Reviews Neuroscience, 7(11), 860-872.

Rayner, K., \& Bertera, J. H. (1979). Reading without a fovea. Science, 206, 468-469.

Rizzolatti, G., Riggio, L., Dascola, I., \& Umilta, C. (1987). Reorienting attention across the horizontal and vertical meridians: evidence in favor of a premotor theory of attention. Neuropsychologia, 25(1A), 31-40.

Schneider, W. X. (1995). VAM: A Neuro-Cognitive Model for Visual Attention Control of Segmentation, Object Recognition, and Spacebased Motor Action. Visual Cognition, 2, 331375.

Schneider, W. X., \& Deubel, H. (2002). Selection-forperception and selection-for-spatial-motor action are coupled by visual attention: A review of recent findings and new evidence from stimulusdriven saccade control. In W. Prinz \& B. Hommel (Eds.), Attention and Performance XIX: Common Mechanisms in Perception and Action (pp. 609-627). Oxford: Oxford University Press.

Schuett, S., Heywood, C. A., Kentridge, R. W., \& Zihl, J. (2008). Rehabilitation of hemianopic dyslexia:
Lingnau, A., Albrecht, T., Schwarzbach, J., \& Vorberg, D. (2014) Visual Search Without Central Vision

are words necessary for re-learning oculomotor control? Brain, 131(Pt 12), 3156-3168.

Seiple, W., Clemens, C., Greenstein, V. C., Holopigian, K., \& Zhang, X. (2002). The spatial distribution of selective attention assessed using the multifocal visual evoked potential. Vision Research, 42(12), 1513-1521.

Seiple, W., Szlyk, J. P., McMahon, T., Pulido, J., \& Fishman, G. A. (2005). Eye-movement training for reading in patients with age-related macular degeneration. Invest Ophthalmol Vis Sci, 46(8), 2886-2896.

Smith, D. T., \& Schenk, T. (2012). The Premotor theory of attention: time to move on? Neuropsychologia, 50(6), 1104-1114.

Sommerhalder, J., Oueghlani, E., Bagnoud, M., Leonards, U., Safran, A. B., \& Pelizzone, M. (2003). Simulation of artificial vision: I. Eccentric reading of isolated words, and perceptual learning. Vision Research, 43, 269283.

Sparks, D. L. (1999). Conceptual issues related to the role of the superior colliculus in the control of gaze. Current Opinion in Neurobiology, 9, 698-707.

Stelmack, J. A., Massof, R. W., \& Stelmack, T. R. (2004). Is there a standard of care for eccentric viewing training? Journal of Rehabilitation Research and Development, 41(5), 729-738.

Thompson, K. G., Biscoe, K. L., \& Sato, T. R. (2005). Neuronal basis of covert spatial attention in the frontal eye field. Journal of Neuroscience, 25(41), 9479-9487.

Timberlake, G. T., Mainster, M. A., Peli, E., Augliere, R. A., Essock, E. A., \& Arend, L. E. (1986). Reading with a macular scotoma. I. Retinal location of scotoma and fixation area. Investigative Ophthalmology and Visual Science, 27, 1137-1147.

Trauzettel-Klosinski, S., \& Brendler, K. (1998). Eye movements in reading with hemianopic field defects: the significance of clinical parameters. Graefe's Archive for Clinical and Experimental Ophthalmology, 236(2), 91-102.

White, J. M., \& Bedell, H. E. (1990). The oculomotor reference in humans with bilateral macular disease. Invest Ophthalmol Vis Sci, 31(6), 11491161. 
Journal of Eye Movement Research

$7(2): 4,1-14$

Zangemeister, W. H., Dannheim, F., \& Kunze, K. (1986). Adaptation of gaze to eccentric fixation in homonymous hemianopia. In E. L. Keller \& D. Zee (Eds.), Adaptive Processes in Visual and Oculomotor Systems (Vol. 57, pp. 247-252). Amsterdam: Elsevier.
Lingnau, A., Albrecht, T., Schwarzbach, J., \& Vorberg, D. (2014) Visual Search Without Central Vision

Zeevi, Y. Y., Peli, E., \& Stark, L. (1979). Study of eccentric fixation with secondary visual feedback. Journal of the Optical Society of America, 69, 669-675. 
Journal of Eye Movement Research $7(2): 4,1-14$
Lingnau, A., Albrecht, T., Schwarzbach, J., \& Vorberg, D. (2014) Visual Search Without Central Vision
Tables

Search time [sec]

\begin{tabular}{ccccc}
\hline & \multicolumn{2}{c}{ FF-L } & \multicolumn{2}{c}{ FF-R } \\
\hline & \multicolumn{2}{c}{ scan path direction } & \multicolumn{2}{c}{ scan path direction } \\
& LR & RL & LR & RL \\
\hline near & $14.3(1.0)$ & $12.1(1.5)$ & $12.8(2.2)$ & $15.3(1.9)$ \\
far & $17.8(1.6)$ & $20.8(3.6)$ & $22.0(4.9)$ & $21.4(3.0)$ \\
\hline
\end{tabular}

Table 1: Search time [sec] as a function of FF location (left, right), scan path direction ( $L R, R L)$ and $F F$ eccentricity (near, far). The standard error of the mean is shown in brackets.

\section{Percentage of errors [\%]}

\begin{tabular}{ccccc}
\hline & \multicolumn{2}{c}{ FF-L } & \multicolumn{2}{c}{ FF-R } \\
\hline & \multicolumn{2}{c}{ scan path direction } & \multicolumn{2}{c}{ scan path direction } \\
& LR & RL & LR & RL \\
\hline near & $29.8(7.3)$ & $16.7(5.6)$ & $24.2(6.2)$ & $27.1(8.0)$ \\
far & $36.9(5.4)$ & $30.4(7.6)$ & $35.6(9.0)$ & $43.2(10.1)$ \\
\hline
\end{tabular}

Table 4: Percentage of errors as a function of FF location (FF-L, FF-R), scan path direction ( $L R, R L)$ and FF eccentricity (near, far). The standard errors of the mean are shown in brackets.

Number of fixations

\begin{tabular}{ccccc}
\hline & \multicolumn{2}{c}{ FF-L } & \multicolumn{2}{c}{ FF-R } \\
\hline & \multicolumn{2}{c}{ scan path direction } & \multicolumn{2}{c}{ scan path direction } \\
& LR & RL & LR & RL \\
\hline near & $39.8(6.6)$ & $38.9(9.3)$ & $40.7(9.3)$ & $40.9(9.5)$ \\
far & $45.7(10.2)$ & $56.6(17.3)$ & $59.5(15.4)$ & $50.7(10.3)$ \\
\hline
\end{tabular}

Table 2: Mean of the number of fixations as a function of $F F$ location (FF-L, FF-R), scan path direction ( $L R, R L)$ and $F F$ eccentricity (near, far). The standard errors of the mean are shown in brackets.

\section{Fixation duration [msec]}

\begin{tabular}{ccccc}
\hline & \multicolumn{2}{c}{ FF-L } & \multicolumn{2}{c}{ FF-R } \\
\hline & \multicolumn{2}{c}{ scan path direction } & \multicolumn{2}{c}{ scan path direction } \\
LR & RL & LR & RL \\
\hline near & $484.7(40.6)$ & $434.9(34.4)$ & $426.8(13.5)$ & $531.0(38.3)$ \\
far & $533.9(44.1)$ & $434.9(34.4)$ & $465.7(29.3)$ & $554.7(47.1)$ \\
\hline
\end{tabular}

Table 3: Fixation duration [msec] as a function of FF location (FF-L, FF-R), scan path direction (LR, RL) and FF eccentricity (near, far). The standard errors of the mean are shown in brackets. 